\title{
25163 - VENTILATORY SUPPORT OF PREGNANT PATIENTS WITH RESPIRATORY DISTRESS SYNDROME
}

\author{
Alexander Loscovich MD, Sorina Grisaru-Granovsky, MD; Moshe Hersch, MD; \\ Michael Schimmel, ; Arnon Samueloff, Pr \\ SWCH, Toronto, ONTARIO, Canada
}

Introduction: Acute lung injury/acute respiratory distress syndrome (ALI/ARDS) in the pregnant patient is a difficult challenge for the critical care and obstetric team. We present our experience with conservative management of pregnant patients with mechanical ventilation secondary to ALI/ARDS, and evaluate the effect of antepartum ventilatory support on the mother and neonate.

Methods: Pregnant women admitted to intensive care units SZMC (Jerusalem, Israel) in 1998-2003 and requiring mechanical ventilation to ALI/ARDS were identified. The medical records were reviewed for demographic date, criteria for intubation, APACHE 2 score, mode of ventilation and outcome. All neonates of these patients were under followed up for physical examination and motor skills.

Results: Sixty nine gravidas required admission to the intensive care (1998-2003), of which 3 mothers suffered of (ALI/ARDS) and required intubation for ALI/ARDS. Intubated mothers delivered 5 fetuses (two sets of twins and one singleton). Criteria for intubation were based on the physician's concern for failing arterial oxygenation and exhaustion. Mechanical ventilation initiated within 24-48 hours of admission. All women were otherwise healthy, mean age 30 years (range 29-32). The range of gestational age at the time of endotracheal intubation was $21-28$ weeks. The ventilatory indices and the ICU data are shown (TABLE). Analgesia/sedation, antibiotics, vassopressors and betamethsone for fetal lung maturity were used in all cases. None received tocolysis. Despite the distended abdomen ventilatory support was maintained according to accepted guidelines (low tidal volume). FiO2 was maintained below critical levels (0.6) and weaned by at least $30 \%$ by the end of the intubation period. The arterial $\mathrm{pH}$ was maintained at $>7.3$. All three women were weaned, discharged with normal respiratory function and subsequently delivered healthy term neonates. Two delivered vaginally (one singleton, one pair of twins) and one by cesarean for obstetric indication. The mean birth weight was 2530 grams (range 2060-3230), and 1'/5'Apgars were above 7. All neonates were healthy and the follow up for a mean of 17 months (range 3-24) was normal.

Conclusions: The outcome of maternal ALI/ARDS is independent of their gravida status and is a function of the underlying cause. Maternal ALI/ARDS is amenable to modern intensive care, thus there is no need to routinely and prematurely terminate pregnancy. One may anticipate that such pregnancies can be safely carried to term. 